\title{
Response to "Limitations in the Hilbert Transform Approach to Locating Solar Cycle Terminators" by R. Booth
}

\author{
Robert J. Leamon ${ }^{1,2}$ (D) Scott W. McIntosh ${ }^{3}$ (D) Sandra C. Chapman ${ }^{4}$ (D) \\ Nicholas W. Watkins ${ }^{4,5,6}$
}

Received: 9 June 2021 / Accepted: 11 September 2021 / Published online: 22 October 2021

(C) The Author(s) 2021

\begin{abstract}
Booth (Solar Phys. 296, 108, 2021; hereafter B21) is essentially a critique of the Hilbert transform techniques used in our paper (Leamon et al., Solar Phys. 295, 36, 2020; hereafter L20) to predict the termination of solar cycles. Here we respond to his arguments; our methodology and parameter choices $d o$ extract a mathematically robust signature of terminators from the historical sunspot record. We agree that the attempt in L20 to extrapolate beyond the sunspot record gives a failed prediction for the next terminator of May 2020, and we identify both a possible cause and remedy here. However, we disagree with the B21 assessment that the likely termination of Solar Cycle 24 is two years after the date predicted in L20, and we show why.
\end{abstract}

Keywords Solar Cycle · Observations

Booth (2021), hereafter B21, is essentially a critique of the Hilbert transform techniques used in our paper (Leamon et al., 2020, hereafter L20) to predict the termination of solar cycles, and by extension, the predictions of the amplitude of Solar Cycle 25 made by (McIntosh et al., 2020, hereafter M20).

As a reminder, a solar cycle "terminator," also referred to as "the termination event" is not Solar Minimum, and it is not the beginning of Solar Cycle 25. Rather, it is the end of Solar Cycle 24, when the magnetic imprint of the old solar cycle finally vanishes from

R.J. Leamon

robert.j.leamon@nasa.gov

1 Goddard Planetary Heliophysics Institute, University of Maryland-Baltimore County, Baltimore, MD 21250, USA

2 NASA Goddard Space Flight Center, Code 672, Greenbelt, MD 20771, USA

3 National Center for Atmospheric Research, High Altitude Observatory, P.O. Box 3000, Boulder, CO 80307, USA

4 Centre for Fusion, Space and Astrophysics, University of Warwick, Coventry CV4 7AL, UK

5 Grantham Research Institute on Climate Change and the Environment, London School of Economics and Political Science, London WC2A 2AE, UK

6 School of Engineering and Innovation, STEM Faculty, Open University, Milton Keynes, MK7 6AA, UK 

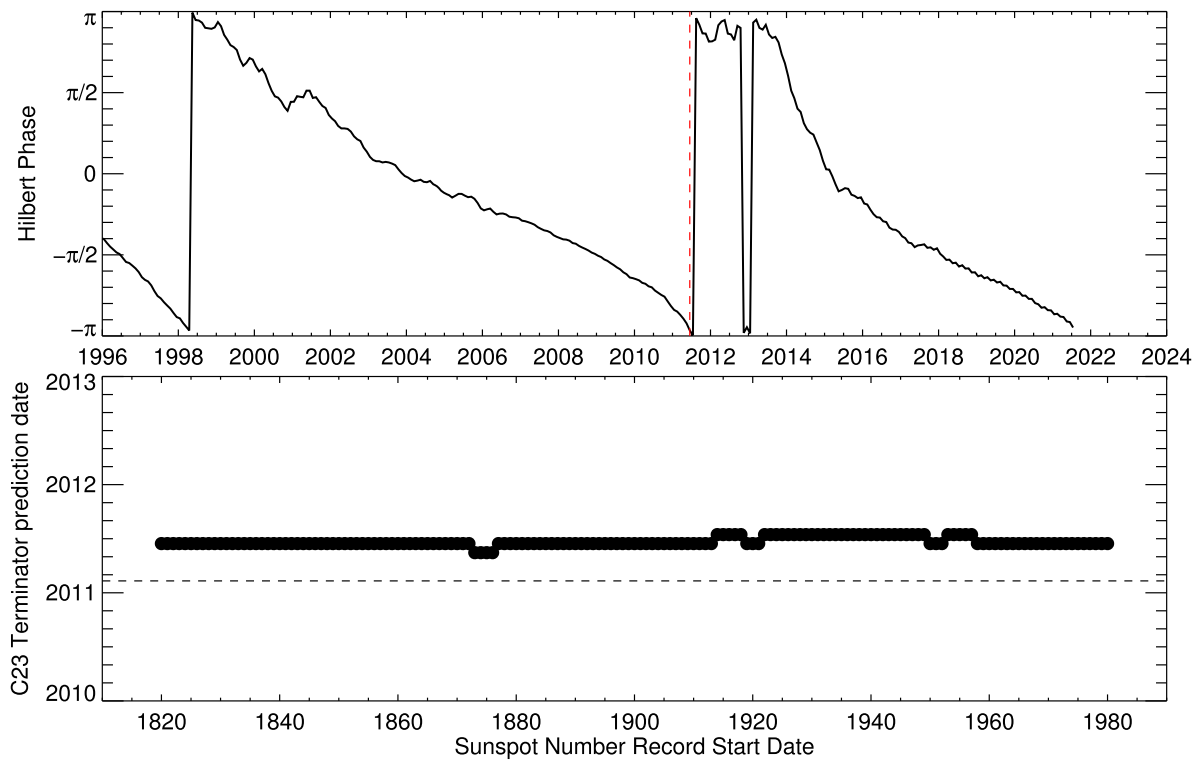

Figure 1 The C23 terminator date is obtained via Hilbert transform of increasingly long time intervals of the SSN record. The dashed horizontal line in the bottom panel indicates the observed date, February 2011.

the Sun's surface. As described by McIntosh et al. (2019), the cancellation of (the last) old cycle polarity at the equator leads to a rapid emergence of a new cycle magnetic flux in mid-latitudes, that is, a rapid growth of sunspot emergence and associated eruptive activity, and a sharp increase in radiative output, most noticeable in coronal Iron lines in the EUV, and the F10.7 solar radio flux.

The termination of Solar Cycle 23 occurred in February 2011. L20 predicted (at date of submission in October 2019) that the termination of Solar Cycle 24 would occur in May 2020 - an extremely short cycle of only 9.25 years. That forecast is clearly premature.

The main points raised by B21 all refer to important features of practically deploying the Hilbert transform on physical time-series that are noisy, of finite length, and not intrinsically zero crossing. These properties of the Hilbert transform are well known and are widely reported in the literature (Boashash, 1992; Pikovsky et al., 2002). Nevertheless, understanding these points is essential in understanding the applicability of the Hilbert transform and its uncertainties, and so we welcome the opportunity to elaborate on them further here.

1. Multiple cycles of the time series are required. In his comment, B21 mistakenly refers to a 1945 (or thereabouts) start date for our Hilbert transform of SSN. In L20, we used a start date of 1820 as shown in Figure 3 of that paper. B21 then finds that the results of the Hilbert transform, thats is, the end date, are indeed sensitive to variations in the start date when 1945 is used. Since a 1945 start date would imply a record extending over only 6-7 cycles (rather than 18 cycles if we go back to 1820), the B21 finding of a sensitivity to the start date is not surprising. However, this does not establish whether 18 cycles are enough to eliminate sensitivity to the start date, so inspired by B21 we address this here. There are two steps to our prediction, (i) the Hilbert transform of the SSN record and (ii) the extrapolation at the edge. Figures 1 and 2 investigate the fidelity of both these steps. In Figure 1, we plot our determination of the occurrence date of the previous terminator as we vary the start date of the SSN record used to perform the Hilbert transform. We can see that the terminator value, 

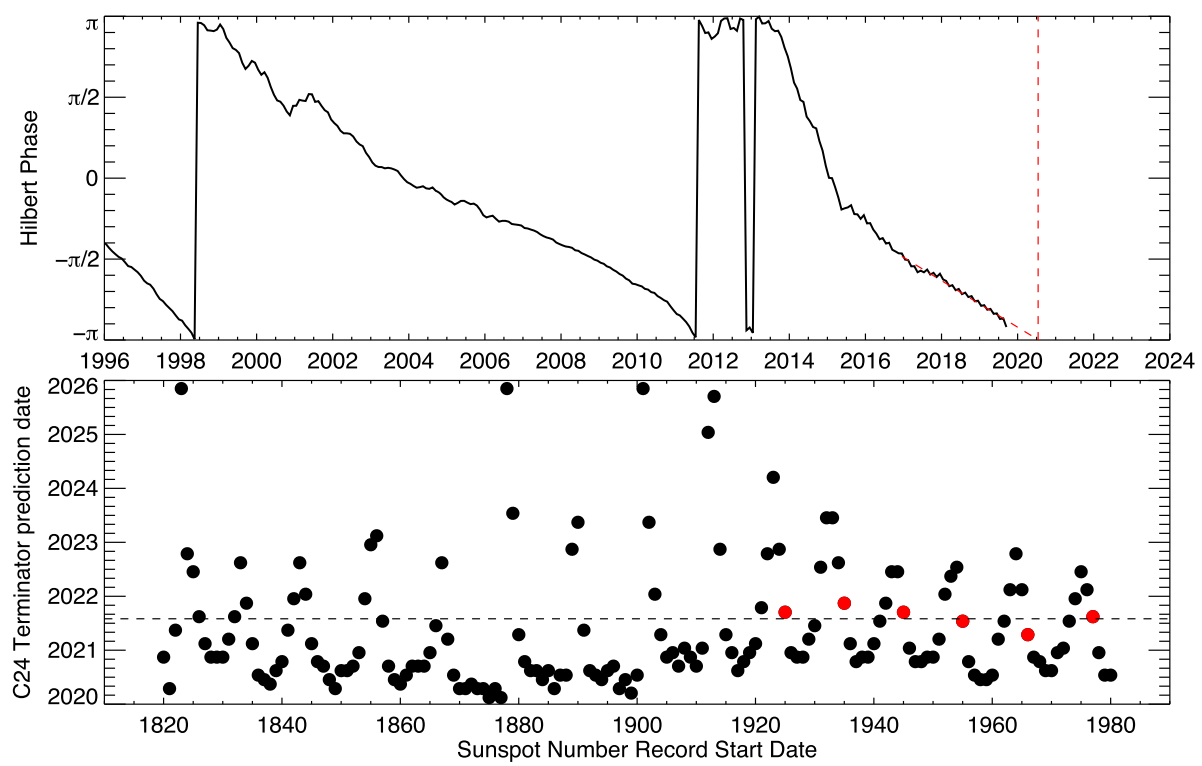

Figure 2 (Top) The original C24 prediction of May 2020, per L20's extrapolation of fit from January 2017 to September 2019. (Bottom) The predicted C24 terminator date is obtained via Hilbert transform of increasingly long time intervals of the SSN record. Note that the predicted date varies with the cycle phase of the start date. A visual inspection suggests that if (as) the Terminator is imminent (the dashed horizontal line of August 2021 in the bottom panel), then the most accurate predictions come from start dates close to the terminators (phase close to $\pm \pi$ ) of other cycles - the terminators of last few cycles are indicated in red.

which is identified as the point where the Hilbert phase intersects $-\pi$ here, is rather robust, even when a quite short SSN record is used. In Figure 2, we plot our prediction for the next terminator as we vary the start date of the SSN record used. This now requires extrapolation at the edge. We can see a clear solar cycle variation in the predicted value; as the SSN record used for the Hilbert transform is successively increased through each solar cycle, it cycles through a systematic bias on the predicted terminator date. This systematic bias can arise as we are estimating the analytic phase using the discrete Hilbert transform (DHT) over a finitelength data interval. The DHT is computed (see Marple, 1999) in a manner that assumes a periodic structure, in the same manner that the discrete Fourier transform approximates the continuous one. If the DHT is performed over an SSN time series that is not a whole number of solar cycles, then a systematic bias will be introduced. This only becomes important close to the edge: in Figure 3, we plot the Hilbert phase time series evaluated with SSN start dates at different points in the solar cycle. Thus values for terminators that have already occurred can robustly be obtained from the DHT without sensitivity to the start (or end) date of the SSN record used. Predicting the next terminator, which requires an extrapolation at the edge, will be subject to this bias. We can minimize this bias by choosing a start date that coincides with a (known) terminator and obtain an uncertainty estimate by generating an ensemble of predictions, which each use a different, known terminator as the SSN start date. Modeling of the systematic bias plotted in Figure 2 may also improve the fidelity of the prediction but is beyond the scope of this Reply.

In L20, we perform a Hilbert transform of the SSN record to obtain a relationship between time and solar cycle phase. This relationship maps the irregular (in time) solar cycle onto a regular $[0,2 \pi]$ interval in phase; effectively, it provides a regular "clock" for the solar 


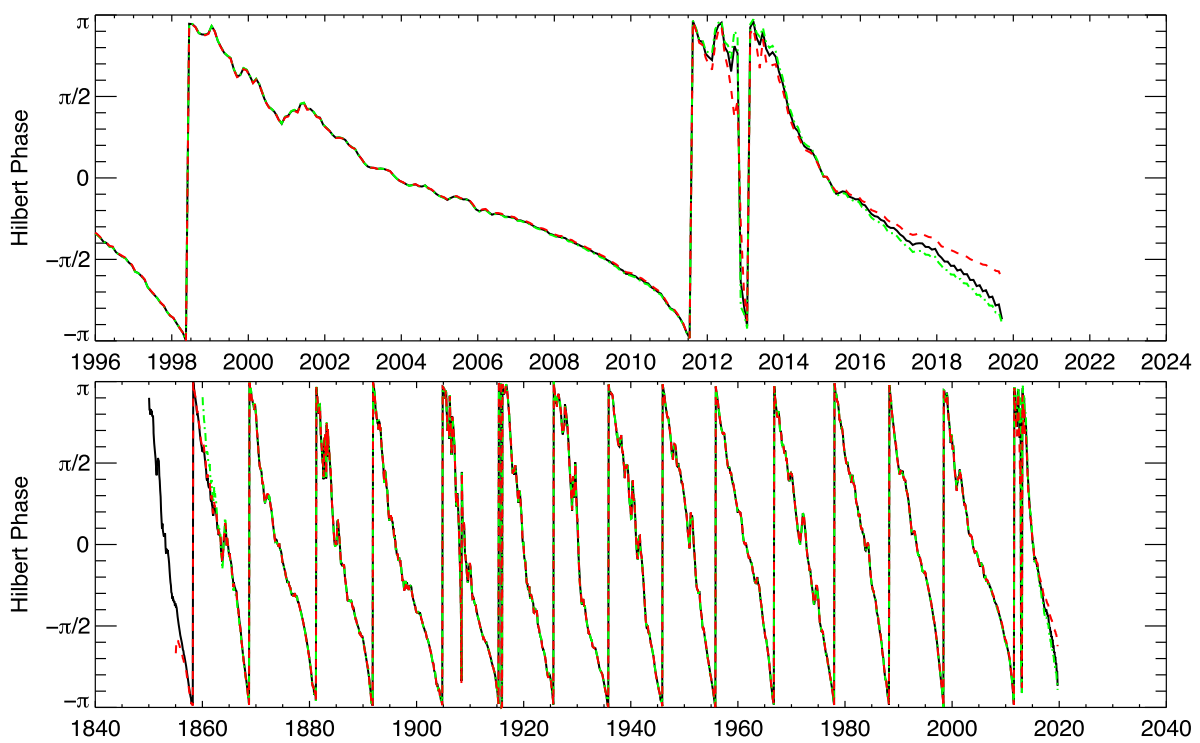

Figure 3 Showing the edge effects of different start dates. Hilbert phase for start dates 1850 (black), 1855 (red), and 1860 (green), for the whole record (bottom) and the last two solar activity cycles (top). Note that the only deviation is in the last $\sim 4$ years, and most pronounced for the red curve, 1855 was close to minimum, 1850 was close to maximum, and 1860 was at maximum.

cycle. Having used the 18 cycles of SSN to obtain this mapping or clock, we can use the same time-to-phase mapping to overplot other quantities on a single clock or epoch (Chapman et al., 2020) to explore solar cycle climatology. These quantities, such as F10.7 and hemispheric sunspot numbers as in L20, may only be available for a few solar cycles, but crucially we do not perform the Hilbert transform directly on them; instead, we use the timephase mapping obtained from the longer SSN record as the single time-to-phase mapping for all compared quantities. In L20 the signature of changes in several different quantities provides information on the timing of the terminators.

2. Signal phase is always relative. The Hilbert transform as its output gives an amplitude and phase that vary in time. The amplitude and phase refer to a zero crossing, oscillatory signal. Before a Hilbert transform can be computed on the SSN, it is therefore necessary to subtract a constant value or slowly time-varying trend. The Hilbert transform is then performed on the background subtracted signal. Considering the subtraction of a constant value $B_{0}$ (referred to in B21 as the "centralizing constant") as is done in L20, the absolute phase obtained from the Hilbert transform will clearly vary with the choice of $B_{0}$ since the value of $B_{0}$ determines the zero crossings of the background subtracted signal. Importantly, the relative phase (between events occurring on successive cycles, i.e., the terminators) will be robust against the choice of $B_{0}$, provided that each cycle is zero crossing; so that each cycle is interpreted by the Hilbert transform as a single transit through $2 \pi$ of phase. To predict when the next terminator will occur, it is the time interval between one terminator and the next that is the required quantity that we seek to determine in L20. In plots of phase versus time, we can set the zero phase to be any convenient value relative to that of the terminators that have already occurred; here in L20, it is set to $-\pi$, and it is noted that this is indeed the phase at which terminators have been seen to occur previously. 


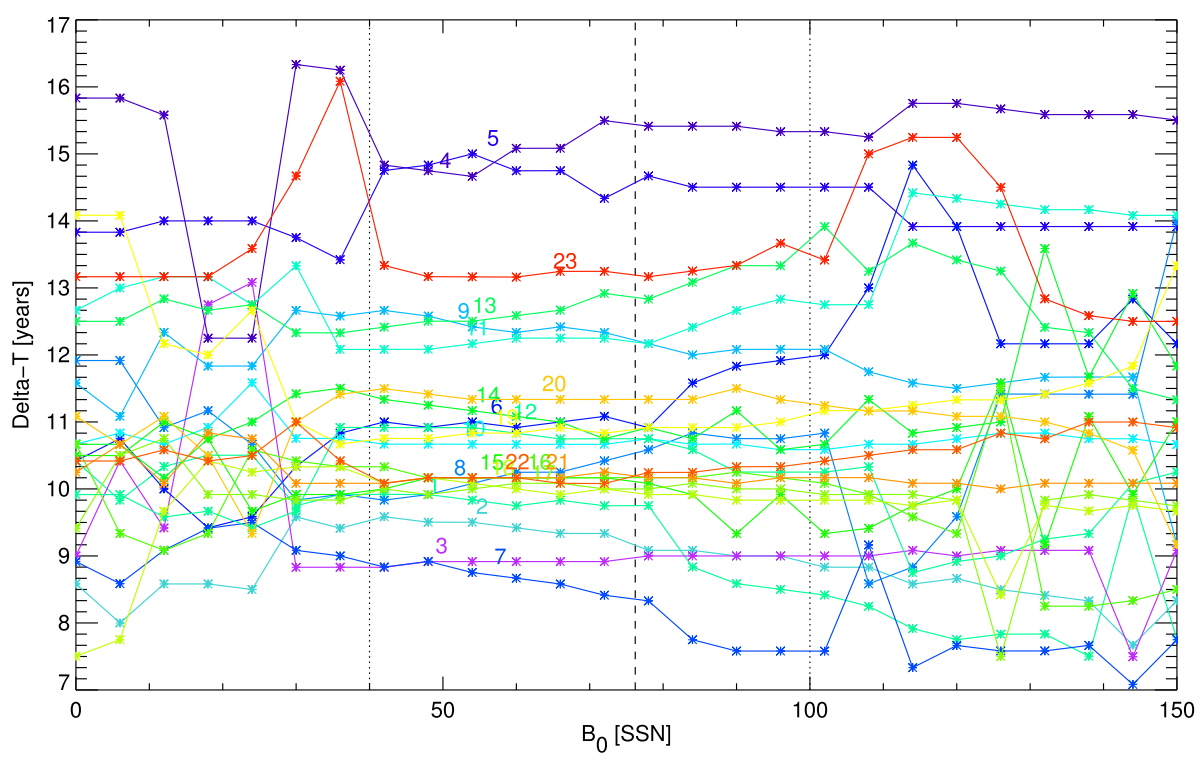

Figure 4 The variation of delta terminators versus $B_{0}$. Each colored line represents (the length of) one solar cycle. There is little variation of $\Delta T\left(=T_{i+1}-T_{i}\right)$ for $B_{0} \simeq 40-100$, as marked by the vertical dotted lines. The dashed vertical line is 76.2, the value used in L20 (mean of SSN 1947-2019). For comparison, the mean of the whole sunspot record from 1749-2021 is 81.7 .

3. Obtained phase differences and their timings are reasonably robust against variation in the "centralizing constant" within a sensible range of values, and this uncertainty can be estimated. Only a purely sinusoidal signal will produce phase differences that are independent of the choice of $B_{0}$. Unavoidably, any real-world signal of interest will be composed of multiple frequencies, and the relative phases of these modes will in part determine when zero crossings occur and hence the specific timings/phases of events such as terminators. However, by repeating the analysis for a range of $B_{0}$ we can estimate the variation in the time intervals between one terminator and the next, and this was done for the analysis in L20; we show the results here in Figure 4. The choice of $B_{0}=76.2$ used in L20 falls within the range of $B_{0}$ for which the delta (terminators) does not change much. This translates into our overall uncertainty estimates.

4. Hilbert transforms incur edge effects. It is well known [e.g., Appendix A2 of Pikovsky et al., 2002] that the result of the Hilbert transform will be less reliable at the edge of a finite-length time series, and the errors will be a systematic drift rather than a random scatter. Global effects have been discussed in point 1 above. We explicitly analyze the local effect of extrapolating beyond the end date in Figure 6 of L20 as noted in B21. We use past cycles where the Hilbert transform can be computed far from the edge of the data to hindcast what this uncertainty may be. However, this remains one of the challenges of forward prediction using this method. We have used a linear extrapolation throughout in L20. Whilst we appreciate, as pointed out by B21, that a nonlinear extrapolation may be more accurate, the difference between linear and nonlinear extrapolation is rather small compared to the systematic error introduced by the edge effects of the Hilbert transform. Of course, as time passes and more data is added, the procedure will become more accurate, and we attempted to quantify this in Figure 6 of L20. We just note that whilst the uncertainty in the 
prediction improves as the event is approached (and then passed), the operational value of the prediction diminishes.

The M20 prediction of $233_{-33}^{+21}$ maximum sunspot number was based on L20's predicted date of 2020.37. It was always our intention to write an update once the terminator occurred. Were it to occur imminently, say in September 2021, Cycle 24 would still be a relatively short cycle at approximately 10.5 years in length, which would give a (downwardly) revised prediction of $\approx 195 \pm 17$. Finally, unlike the conclusion of B21, it is improbable that the Cycle 24 terminator will occur (more than) two years after the date predicted in L20, the average minimum-terminator separation is $23 \pm 5$ months, and we are already almost at 23 months from the SIDC-proclaimed date of December 2019.

\section{Declarations}

Disclosure of Potential Conflicts of Interest The authors declare that they have no conflicts of interest.

Open Access This article is licensed under a Creative Commons Attribution 4.0 International License, which permits use, sharing, adaptation, distribution and reproduction in any medium or format, as long as you give appropriate credit to the original author(s) and the source, provide a link to the Creative Commons licence, and indicate if changes were made. The images or other third party material in this article are included in the article's Creative Commons licence, unless indicated otherwise in a credit line to the material. If material is not included in the article's Creative Commons licence and your intended use is not permitted by statutory regulation or exceeds the permitted use, you will need to obtain permission directly from the copyright holder. To view a copy of this licence, visit http://creativecommons.org/licenses/by/4.0/.

\section{References}

Boashash, B.: 1992, Estimating and interpreting the instantaneous frequency of a signal. I - Fundamentals. II - Algorithms and applications. Proc. IEEE 80, 520. ADS.

Booth, R.J.: 2021, Limitations in the Hilbert transform approach to locating solar cycle terminators. Solar Phys. 296, 108. DOI. ADS.

Chapman, S.C., McIntosh, S.W., Leamon, R.J., Watkins, N.W.: 2020, Quantifying the solar cycle modulation of extreme space weather. Geophys. Res. Lett. 47, e87795. DOI. ADS.

Leamon, R.J., McIntosh, S.W., Chapman, S.C., Watkins, N.W.: 2020, Timing terminators: forecasting Sunspot Cycle 25 onset. Solar Phys. 295, 36. DOI. ADS.

Marple, S.L.: 1999, Computing the discrete-time "analytic" signal via FFT. IEEE Trans. Signal Process. 47, 2600. DOI. ADS.

McIntosh, S.W., Leamon, R.J., Egeland, R., Dikpati, M., Fan, Y., Rempel, M.: 2019, What the death of solar cycles imply about the nature of the solar interior. Solar Phys. 294, 88. DOI.

McIntosh, S.W., Chapman, S., Leamon, R.J., Egeland, R., Watkins, N.W.: 2020, Overlapping magnetic activity cycles and the sunspot number: forecasting Sunspot Cycle 25 amplitude. Solar Phys. 295, 163. DOI. ADS.

Pikovsky, A., Rosenblum, M., Kurths, J., Hilborn, R.C.: 2002, Synchronization: a universal concept in nonlinear science. Am. J. Phys. 70, 655. DOI. ADS.

Publisher's Note Springer Nature remains neutral with regard to jurisdictional claims in published maps and institutional affiliations. 